\title{
Trauma systems and emergency medical services: The missing link for tranexamic acid utilization in major trauma
}

\author{
John M. Tallon, MD, MSc ${ }^{* \dagger \neq}$
}

Trauma remains a common and continuing clinical challenge for all emergency physicians and associated healthcare providers across all facilities and regions from rural/remote emergency departments to large urban emergency departments. ${ }^{1}$ With a 26 billion dollar annual price tag, trauma represents a major preventable economic and health issue for Canadians, ${ }^{2}$ yet this disease process is still often neglected in acute care health literature. Trauma is also a neglected disease in the context of associated dollars invested in research. Trauma research receives the lowest funding relative to the burden of morbidity and mortality that it creates when compared with all other diseases. ${ }^{3}$

Injuries are the leading cause of death for Canadians between the ages of 1 to 44 years, and the third leading cause of death for Canadians ages 45 to 64 years. $^{4}$ Moreover, injuries are among the top causes of hospitalizations for Canadians of all ages. ${ }^{5}$ Trauma remains the most common cause of potential life years lost and disability adjusted life years and has a significant impact on individuals, their families, and their communities. ${ }^{3}$ Trauma, in its most dramatic resuscitative acute phase, has outcomes directly tied to the application and timing of specific interventions applied by healthcare professionals, including airway interventions, blood products, pro-coagulants, bleeding control, optimization of hemodynamics, rapid transport (to definitive care), and timely surgical intervention as indicated. ${ }^{6}$

In the context of trauma acute care, I will specifically focus this editorial on the paper by Ghawnni et al. on the evaluation of the utilization of life-saving tranexamic acid (TXA) in a tertiary trauma centre. ${ }^{7}$

In this observational study, the authors from the trauma program at Hamilton Health Sciences Centre and McMaster University bravely report on a retrospective evaluation of a cohort of major trauma patients and compliance with TXA administration. Their inclusion criteria are those parameters defined in the CRASH-2 trial for the administration of TXA, ${ }^{8}$ combined with those inclusion criteria used in the MATTERS trial, ${ }^{9}$ an observational study that demonstrated a mortality benefit in major trauma patients receiving TXA. I say "bravely report" because their published results are less than exemplary, with a compliance rate of $27.1 \%$, hardly a number that defines cutting edge, evidenced informed tertiary (or other) care practice. They also note that they are the first to perform this audit and publish it. By publishing these results, I hope they will stimulate other trauma centres to carefully review their own performance metrics for TXA administration. Looking ahead, it is possible that this metric or index may ultimately become part of the expected requirements for future Trauma Distinction accreditation metrics through Accreditation Canada. ${ }^{10}$

The authors note that hemorrhage resulting from vascular disruption remains the leading cause of preventable death in civilian trauma. ${ }^{3,7}$ They also note that the CRASH-2 trial recommends the use of TXA within 3 hours of injury. ${ }^{8}$ They excluded from their analysis those patients who received TXA at other sending sites (21 of 534 patients), and their final cohort for analysis, after excluding patients with missing data, was 495 patients over a 2-year period. 9

The authors report that their mean time to administration of TXA was 47 minutes, measured from the

From the *British Columbia Emergency Health Services, British Columbia, Vancouver, BC; †Department of Emergency Medicine, University of British Columbia, Vancouver, BC; and ‡Departments of Emergency Medicine, Community Health and Epidemiology, Anesthesia, and Surgery, Dalhousie University, Halifax, NS.

Correspondence to: Dr. John Tallon, 150 - 2955 Virtual Way, Vancouver, BC V5M 4X6; Email: john.tallon@bcehs.ca

(C) Canadian Association of Emergency Physicians

CJEM 2018;20(2):167-169

DOI 10.1017/cem.2018.22 
patient's hospital arrival time in the group $(27.1 \%$, $\mathrm{n}=134$ ) who actually received TXA. ${ }^{7}$ However, no effort was made to determine and report the total actual time from injury to drug administration. They assume that their time of 47 minutes represents a good, CRASH-2 compliant metric because this surrogate measurement is within 3 hours of injury. However, this time metric is insufficient in the context of actually knowing the time of injury, which should be available through the ambulance/paramedic patient care record.

The authors appropriately lament their poor overall compliance with this readily available, inexpensive, evidence-informed medication, which is now listed on the World Health Organization (WHO) essential medication list. ${ }^{11}$ They offer reasons for non-compliance, which include inadequate in-house knowledge translation and implementation, the lack of a formal in-house guideline, and lack of formal associated educational initiatives. Also, although I agree with this assessment, I also believe there is a further explanation that would need to be addressed to approach $100 \%$ compliance and to achieve optimal patient outcomes.

For these goals to be met, the entire trauma system must be used; one needs to go further upstream beyond in-hospital initiatives to make this medication administration successful. This medication should be given by the first emergency medical services (EMS) personnel to arrive on the scene; it should be given in the context of all of the indications currently listed in Ghawnni et al.'s paper, but, by paramedics at the scene, as close to time of injury as possible rather than waiting until arrival at the hospital. ${ }^{12}$ Furthermore, its use must be protocolized and closely monitored through clinical oversight. If this is done, there will be no $27.1 \%$ compliance rate at the hospital, but rather a near $100 \%$ compliance rate (unless, perhaps, some bleeding, dying patients are delivered directly to the emergency department by a private vehicle, and the TXA intervention is subsequently missed).

Not only can TXA be given by EMS, but also it is being given by $\mathrm{EMS},{ }^{13}$ and its associated evidence virtually demands that this practice be taken up in the context of time of injury, potential long-distance transports, interfacility transports, and that its impact is highest when closest to the time of injury. ${ }^{14}$, In fact, two further studies may widen indications for timely TXA use. These studies are the Resuscitation Outcomes Consortium (ROC) study of TXA in traumatic brain injury (TBI), ${ }^{15}$ which is at the analysis stage, and the
CRASH-3 trial (still recruiting), which is also being conducted to assess the effect of TXA on risk of death or disability in patients with TBI. ${ }^{16}$ These two studies may elucidate evidence that the clinical use of TXA in trauma be expanded, an even more important potential reason for its use to be implemented in EMS practice. Treatment delay simply cannot be tolerated, as evidenced and discussed recently in a meta-analysis on the use of TXA in an acute severe hemorrhage. ${ }^{17}$

Again, I laud the authors for performing and publishing this study. I believe that the critical solution in the reported poor compliance lies in the optimized role of EMS administering TXA as part of the entire integrated trauma system. My sincere hope is that, by publishing their audit, the authors may inform a new, key metric in trauma care for performance evaluation and accreditation.

Keywords: trauma, TXA, tranexamic acid, compliance, emergency medical services, EMS

Acknowledgements: Special thanks to Dr. Steve Wheeler and Corinne C. Tallon for their careful review of the manuscript.

Competing interests: None declared.

\section{REFERENCES}

1. DiMaggio CJ, Avraham JB, Lee DC, et al. The epidemiology of emergency department trauma discharges in the United States. Acad Emerg Med 2017;24(10):1244-56.

2. Parachute. The cost of injury in Canada; 2015. Available at: http://www.parachutecanada.org/research/item/economicburden-of-injury-reports (accessed 29 January 2018).

3. Spinella PC. Zero preventable deaths after traumatic injury: an achievable goal. 7 Trauma Acute Care Surg 2017; 82(6S Suppl 1):S2-8.

4. Public Health Agency of Canada. Table: leading causes of death, Canada, 2008, males and females combined, counts (age-specific death rate per 100,000); 2013. Available at: https:// www.canada.ca/en/public-health/services/reports-publications/ leading-causes-death-hospitalization-canada/2008-males-femalescombined-counts-specific-death-rate.html (accessed 29 January 2018).

5. Public Health Agency of Canada. Table: leading causes of hospitalizations, Canada, 2008, males and females combined, counts (age-specific hospitalization rate per 100,000); 2013. Available at: https://www.canada.ca/en/ public-health/services/reports-publications/leading-causesdeath-hospitalization-canada.html (accessed 29 January 2018).

6. Cannon JW. Hemorrhagic shock. N Engl 7 Med 2018;378 (4):370-9; doi:10.1056/NEJMra1705649.

7. Ghawnni A, Coates A, Owen J. Compliance of tranexamic acid administration to trauma patients at a level-one trauma centre. CFEM 2017;4:1-6. 
8. CRASH-2 Trial Collaborators. Effects of tranexamic acid on death, vascular occlusive events, and blood transfusion in trauma patients with significant haemorrhage (CRASH-2): a randomised, placebo-controlled trial. Lancet 2010;376(9734): 23-32.

9. Morrison JJ, Dubose JJ, Rasmussen TE, Midwinter MJ. Military application of tranexamic acid in trauma emergency resuscitation (MATTERS) study. Arch Surg 2012;147(2): 113-9.

10. Accreditation Canada. Trauma distinction. Available at: https://accreditation.ca/trauma-distinction/ (accessed 29 January 2018).

11. World Health Organization. WHO model list of essential medicines; 2017. Available at: http://www.who.int/medi cines/publications/essentialmedicines/20th_EML2017_FINAL_ amendedAug2017.pdf?ua=1 (accessed 29 January 2018).

12. Spinella PC, Cap AP. Prehospital hemostatic resuscitation to achieve zero preventable deaths after traumatic injury. Curr Opin Hematol 2017;24(6):529-35.
13. British Columbia Ambulance Service. Treatment guidelines: trauma management; 2016. Available at: https://bctg.bcas. ca/Condition/Interventions/16 (accessed 29 January 2018).

14. Vu EN, Schlamp RS, Wand RT, et al. Prehospital use of tranexamic acid for hemorrhagic shock in primary and secondary air medical evacuation. Air Med 7 2013;32(5): 289-92.

15. London School of Hygiene \& Tropical Medicine. CRASH-3. Available at: http://crash3.lshtm.ac.uk/ (accessed 29 January 2018).

16. Resuscitation Outcomes Consortium. Current studies. Available at: https://roc.uwctc.org/tiki/tiki-index.php?page= current-studies (accessed 29 January 2018).

17. Gayet-Ageron A, Prieto-Merino D, Ker K, et al; Antifibrinolytic Trials Collaboration. Effect of treatment delay on the effectiveness and safety of antifibrinolytics in acute severe haemorrhage: a meta-analysis of individual patient-level data from 40,138 bleeding patients. Lancet 2018;391(10116): 125-32; doi:10.1016/S0140-6736(17)32455-8. 